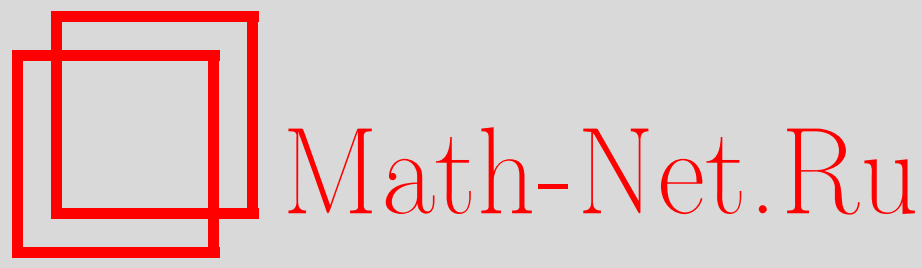

О. В. Подольская, Сложность реализации симметрических булевых функций схемами в базисе антицепных функций, Дискрет. матем., 2015, том 27, выпуск 3, 95107

DOI: https://doi.org/10.4213/dm1337

Использование Общероссийского математического портала Math-Net.Ru подразумевает, что вы прочитали и согласны с пользовательским соглашением http://www . mathnet.ru/rus/agreement

Параметры загрузки:

IP: 3.89 .185 .249

26 апреля 2023 г., 13:49:47

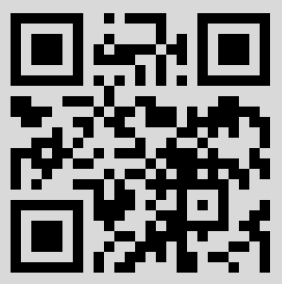




\title{
Сложность реализации симметрических булевых функций схемами в базисе антицепных функций
}

\author{
() 2015 г. О. В. Подольская*
}

Изучается сложность реализации булевых функций схемами из функциональных элементов в бесконечном базисе, состоящем из всех характеристических функций антицепей на булевом кубе. Установлено точное значение сложности реализации произвольной симметрической функции схемами в этом базисе. В частности, для функций четности и голосования от $n$ переменных при всех натуральных $n \geqslant 1$ получены точные значения сложности: $\left\lfloor\frac{n+1}{2}\right\rfloor$ и $\left\lfloor\frac{n}{2}\right\rfloor+1$ соответственно. Установлено, что наибольшая сложность булевых функций от $n$ переменных при реализации схемами в этом базисе по порядку роста равна $n$.

Работа выполнена при поддержке Российского фонда фундаментальных исследований, проект 14-01-00598.

Ключевые слова: сложность булевых функций, антицепные функции, булевы схемы, симметрические булевы функции, функция Шеннона.

\section{1. Введение}

Функция называется симметрической, если она не изменяется при любой перестановке своих переменных. Всякая симметрическая булева функция $f$ от $n$ переменных определяется двоичным набором $\left(a_{0}, a_{1}, \ldots, a_{n}\right)$, где $a_{i}=1$ тогда и только тогда, когда функция $f$ равна 1 на наборах с $i$ единицами. Для симметрической булевой функции $f$ через $k(f)$ будем обозначать количество индексов $i$, для которых $a_{i}$ равны 1. Далее, говоря о симметрических функциях, мы будем иметь в виду симметрические булевы функции.

Булева функция $p_{n}\left(x_{1}, \ldots, x_{n}\right)=x_{1}+\ldots+x_{n}(\bmod 2)$ называется функцией четности. Булева функция $m_{n}\left(x_{1}, \ldots, x_{n}\right)$, принимающая значение 1 на тех и только тех наборах, в которых число единиц не меньше $\frac{n}{2}$, называется функцией голосования.

Булев куб $\{0,1\}^{n}$ рассматривается как частично упорядоченное множество наборов с естественным порядком декартова произведения (при $0 \leqslant 1$ ). Антищепъю на булевом кубе называется всякое подмножество булева куба, состоящее из попарно несравнимых наборов. Булева функция, принимающая значение 1 лишь на некоторой антицепи, называется антицепной. Множество всех антицепных функций

* Место работы: МГУ им. М. В. Ломоносова, e-mail: olgavikonov@gmail.com 
будем обозначать через $A C$ (см. [1]). Это множество замкнуто относительно операций подстановки констант и отождествления переменных, и всякая булева функция выражается через функции из множества $A C$ с помощью операции суперпозиции.

Пусть две действительнозначные функции $a(n)$ и $b(n)$ натурального аргумента принимают положительные значения при всех достаточно больших $n$. Будем говорить, что порядок роста функции $a(n)$ не больше $b(n)$ и обозначать это через $a(n)=O(b(n))$, если существует такая положительная постоянная $c$, что $a(n) \leqslant c b(n)$ при всех достаточно больших $n$. При этом будем говорить, что порядок роста функции $b(n)$ не меньше $a(n)$, и обозначать это через $b(n)=\Omega(a(n))$. Если одновременно $a(n)=\Omega(b(n))$ и $a(n)=O(b(n))$, то будем говорить, что порядок роста $a(n)$ равен $b(n)$, и обозначать это через $a(n)=\Theta(b(n))$.

В работе рассматривается реализация симметрических функций схемами из функциональных элементов в бесконечном базисе $A C$. Определение схемы и других используемых в работе понятий см., например, в [2].

Сложностъю схемы называется число элементов в этой схеме. Сложностью функции называется наименьшая сложность схемы в базисе $A C$, реализующей эту функцию. Будем использовать следующие обозначения: $L(S)$ - сложность схемы $S$, $L(f)$ - сложность функции $f$. Функцией Шеннона называется функция $L(n)=$ $\max L(f)$, где максимум берется по всем булевым функциям $f$, зависящим от $n$ переменных.

В [1], [3], [4] изучались нижние оценки сложности схем в базисе $A C$. В [1] была доказана оценка $\Omega\left(n^{1 / 3}\right)$ сложности функции четности от $n$ переменных. Тем самым также была установлена нижняя оценка функции Шеннона: $L(n)=\Omega\left(n^{1 / 3}\right)$. Далее в [3] была получена оценка $\Omega\left((n / \ln n)^{1 / 2}\right)$ сложности функции четности и функции Шеннона. Развитие метода из [1] позволило улучшить последний результат, и в [4] была доказана оценка $\Omega(\sqrt{n})$ сложности функции четности, функции голосования и почти всех функций от $n$ переменных. Таким образом, в базисе $A C$ было установлено соотношение $L(n)=\Omega(\sqrt{n})$.

В разделе 3 статьи получено точное значение сложности реализации произвольной симметрической функции схемами в базисе $A C$.

Теорема 1. Для любого натурального $n$ и для произвольной симметрической функции $f\left(x_{1}, \ldots, x_{n}\right)$ имеет место равенство

$$
L(f)=\min (k(f), n-k(f)+2) .
$$

С использованием теоремы 1 нетрудно установить точные значения сложности реализации функции четности и функции голосования от $n$ переменных схемами в базисе $A C$.

Теорема 2. Для любого натурального $n$ имеют место равенства

$$
L\left(p_{n}\right)=\left\lfloor\frac{n+1}{2}\right\rfloor, L\left(m_{n}\right)=\left\lfloor\frac{n}{2}\right\rfloor+1 .
$$

Доказательство этого результата изложено в разделе 4.

Что касается верхних оценок, в [1] указана оценка функции Шеннона $L(n) \leqslant n+1$ для всех натуральных $n$. В [5] установлена верхняя оценка $L(n) \leqslant n$ и коротко 
изложены основные идеи доказательства. Эта оценка и нижняя оценка, вытекающая из теоремы 2, в совокупности устанавливают порядок роста функции Шеннона в базисе $A C$.

Теорема 3. В базисе антищепных функиий $L(n)=\Theta(n)$.

\section{2. Некоторые определения и обозначения}

Множество чисел $\{1,2, \ldots, n\}$ будем обозначать через $[n]$.

Обозначим через $\boldsymbol{x}$ произвольный набор значений аргументов $\left(x_{1}, \ldots, x_{n}\right)$. Максимальный набор куба $\{0,1\}^{n}-$ набор $\mathbf{1}=(1, \ldots, 1)$ - будем называть верхним набором куба, а минимальный $-\mathbf{0}=(0, \ldots, 0)$ нижним. Аналогично для всякого подкуба меньшей размерности верхним и нижним наборами будем называть соответственно максимальный и минимальный наборы этого подкуба.

Для любого $P \subseteq[n]$ через $\boldsymbol{x}^{P}$ обозначим такой двоичный набор $\boldsymbol{x}=\left(x_{1}, \ldots, x_{n}\right)$, что для любого $p \in[n]$ условие $x_{p}=1$ выполняется тогда и только тогда, когда $p \in P$.

Множество наборов булева куба $\{0,1\}^{n}$, содержащих ровно $t$ единиц, где $t \in\{0,1, \ldots, n\}$, называется слоем с номером $t$. Ясно, что каждый слой является антицепью на булевом кубе. В этих терминах носитель симметрической функции $f\left(x_{1}, \ldots, x_{n}\right)$, которая равна 1 на наборах, содержащих $i_{1}, \ldots, i_{k(f)}$ единиц, есть объединение слоев с номерами $i_{1}, \ldots, i_{k(f)}$.

Схема из функциональных элементов называется приведенной, если различные входы каждого элемента присоединены к различным вершинам схемы, а на выходе каждого элемента реализуется функция, отличная от константы (см. [3]). Отметим, что для любой схемы в базисе $A C$ сложности $s$, реализующей функцию, отличную от константы, существует приведенная схема в базисе $A C$ сложности не больше $s$, реализующая ту же функцию.

Нумерация элементов схемы называется правилъной, если на входы каждого элемента могут подаваться выходы элементов с меньшими номерами или входы схемы. Известно (см. [6]), что такую нумерацию можно задать в любой схеме (возможно, несколькими способами).

Всюду далее в доказательстве нижних оценок будем рассматривать приведенные схемы в базисе $A C$ с произвольно фиксированной правильной нумерацией элементов.

Элемент схемы с номером $l$ обозначается через $e_{l}$, антицепная функция, которая ему соответствует - через $g_{l}$, а функция, которая реализуется на выходе элемента $e_{l}$ - через $h_{l}$. Значение функции $h_{l}$ при подаче на входы схемы набора $\boldsymbol{x}$ обозначается через $h_{l}(\boldsymbol{x})$. Символы переменных $x_{1}, x_{2}, \ldots, x_{n}$, которые приписаны $n$ входам схемы, будем называть входными переменными схемы.

\section{3. Доказательство теоремы 1}

Докажем две леммы.

4 Дискретная математика, т. 27 № 3 
Лемма 1. Для любого натуралъного $п$ и для произвольной симметрической функции $f\left(x_{1} \ldots, x_{n}\right)$ справедлива оченка $L(f) \geqslant \min (k(f), n-k(f)+2)$.

Лемма 2. Для любого натуралъного $п$ и для произвольной симметрической функции $f\left(x_{1} \ldots, x_{n}\right)$ справедлива оченка $L(f) \leqslant \min (k(f), n-k(f)+2)$.

Вначале в п. 3.1 мы коротко опишем идею доказательства леммы 1, а затем в пп. 3.2, 3.3 изложим подробное полное рассуждение. Далее в п. 3.4, используя идеи из [5], мы докажем лемму 2. Доказательства лемм 1 и 2 в совокупности дадут доказательство теоремы 1.

3.1. Идея доказательства леммы 1. Мы изложим здесь идею доказательства нижней оценки из леммы 1 на примере частного случая. Для произвольной симметрической функции $f$ рассмотрим все схемы, которые реализуют эту функцию и обладают следующим свойством: на входы любого элемента схемы подаются все входы схемы. (В п. 3.4 будет показано, что всякую симметрическую функцию можно реализовать схемой такого вида.) Для любой схемы $S_{f}$ с описанным свойством покажем, что нижняя оценка ее сложности имеет вид $L\left(S_{f}\right) \geqslant k(f)$. Данный частный случай удобен, чтобы продемонстрировать идею доказательства леммы 1 грубо, в самых общих чертах. В общем случае, при доказательстве леммы 1 в пп. 3.2, 3.3, описанное свойство схем, разумеется, не предполагается.

В рассматриваемом частном случае введем понятие первого ненулевого элемента на данном наборе. Пусть при подаче на входы схемы произвольного набора $\boldsymbol{\alpha} \in$ $\{0,1\}^{n}$ элементы $e_{i_{1}}, \ldots, e_{i_{l}}$ есть все такие элементы схемы $S_{f}$, что $h_{i_{j}}(\boldsymbol{\alpha})=1$ для всех $j \in[l]$. Тогда элемент $e_{m}$, обладающий минимальным номером среди элементов $e_{i_{1}}, \ldots, e_{i_{l}}$, будем называть первым ненулевым элементом на наборе $\boldsymbol{\alpha}$. Отметим, что это определение соответствует интуитивному представлению.

Возьмем произвольную цепь $\widetilde{C}$, состоящую из $n+1$ различных наборов куба $\{0,1\}^{n}$. Цепь $\widetilde{C}$ обладает следующим свойством: никакой элемент схемы $S_{f}$ не является дважды первым ненулевым на наборах этой цепи. Действительно, предположим обратное: некоторый элемент $e_{t}$ является первым ненулевым на наборах $\boldsymbol{\beta}$ и $\boldsymbol{\gamma}$ цепи $\widetilde{C}$. Без ограничения общности будем считать, что набор $\boldsymbol{\beta}$ меньше набора $\gamma$. По определению первого ненулевого элемента, все элементы с номерами меньше $t$ выдают 0 на наборах $\boldsymbol{\beta}$ и $\boldsymbol{\gamma}$. Таким образом, получаем, что антицепная функция $g_{t}$, которая соответствует элементу $e_{t}$, выдает 1 на некоторой паре сравнимых наборов. Это противоречит тому, что функция $g_{t}-$ антицепная.

Из доказанного свойства цепи следует, что сложность схемы $S_{f}$ не меньше количества наборов этой цепи, на которых в схеме есть первый ненулевой элемент. Заметим, что если функция $f$ выдает 1 на каком-то наборе, то в схеме $S_{f}$ есть первый ненулевой элемент на этом наборе. Отсюда и вытекает нижняя оценка сложности схемы: $L\left(S_{f}\right) \geqslant k(f)$.

При доказательстве свойства цепи используется тот факт, что схема $S_{f}$ имеет особый вид: на входы элемента $e_{t}$, которому соответствует функция $g_{t}$, непосредственно подаются все входы схемы. В общем случае схема не обладает указанным свойством, и аналогичное утверждение не получится доказать для произвольно выбранной цепи; потребуется построить специальную цепь, следуя определенному правилу. Кроме того, в общем случае понятие первого ненулевого элемента будет 
определено несколько более сложным образом. Указанные технические изменения позволят доказать лемму 1 в общем случае, опираясь на изложенные выше основные идеи.

3.2. Основная конструкция. Доказательство леммы 1. Рассмотрим произвольную симметрическую функцию $f\left(x_{1}, \ldots, x_{n}\right)$, которая равна 1 на $k(f)$ различных слоях куба $\{0,1\}^{n}$. Рассмотрим произвольную схему $S$ в базисе $A C$, реализующую функцию $f$. Пусть $L(S)=s$ и $e_{1}, \ldots, e_{s}$ - все элементы схемы. Элементам приписаны соответственно антицепные функции $g_{1}, \ldots, g_{s}$. Докажем оценку $s \geqslant \min (k(f), n-k(f)+2)$.

Для доказательства построим цепь $C$, состоящую из $n+1$ различных наборов куба $\{0,1\}^{n}$. В качестве крайних наборов цепи $C$ возьмем верхний и нижний наборы куба: $\mathbf{0}, \mathbf{1} \in\{0,1\}^{n}$. Оставшаяся часть п. 3.2 посвящена построению промежуточных наборов цепи.

Говоря неформально, промежуточные наборы цепи мы будем получать, двигаясь по кубу с двух сторон: спускаясь от верхнего набора или поднимаясь от нижнего. «Спуск» от верхнего набора осуществляется так: по определенному алгоритму в текущем наборе выбирается одна из единичных компонент, значение которой изменяется на 0. Полученный набор добавляется в цепь. Значение входной переменной, соответствующей выбранной компоненте, далее считается зафиксированным значением 0 и более не изменяется. Аналогично осуществляется «подъем» от нижнего набора: значение определенной нулевой компоненты текущего набора изменяется на 1, и, тем самым, получается очередной набор цепи; соответствующая входная переменная фиксируется значением 1. Всякий раз при фиксировании значения очередной входной переменной происходит переход к подкубу размерности на единицу меньше, и далее рассматриваются его верхний и нижний наборы.

Вернемся к подробному описанию процесса построения цепи. Чтобы построить цепь, будем рассматривать элементы схемы $S$ в порядке заданной правильной нумерации, начиная с элемента $e_{1}$. Процесс построения будет характеризоваться следующими параметрами.

- Номер шага $i \in[s]$; он определяется номером элемента схемы, с которого начинается данный шаг.

- Одновременно с цепью строятся два множества: $F_{i}, T_{i} \subseteq[n]$. Это множества номеров тех входных переменных схемы, значения которых после $i$-го шага зафиксированы нулями $\left(F_{i}\right)$ и единицами $\left(T_{i}\right)$ соответственно.

Входную переменную схемы будем называть свободной относительно множества $A$, где $A \subseteq[n]$, если номер этой переменной принадлежит множеству $[n] \backslash A$. В процессе построения цепи мы будем рассматривать свободные переменные относительно множеств вида $F_{i} \cup T_{i}:$ неформально, это те переменные, значения которых в определенный момент времени еще не зафиксированы значениями 0 или 1. Входы схемы будем называть свободными относительно множества $A$, где $A \subseteq[n]$, если этим входам приписаны переменные, свободные относительно множества $A$.

На $i$-м шаге рассматривается подкуб с верхним и нижним наборами $\boldsymbol{x}^{[n] \backslash F_{i-1}}$ и $\boldsymbol{x}^{T_{i-1}}$ и строится новый подкуб - с верхним и нижним наборами $\boldsymbol{x}^{[n] \backslash F_{i}}$ и $\boldsymbol{x}^{T_{i}}$ 
- $E_{i}-$ множество таких элементов схемы из множества $\left\{e_{1}, \ldots, e_{i}\right\}$, на входы которых могут подаваться только выходы элементов с меньшими номерами и входы схемы, соответствующие входным переменным с номерами из множества $F_{i} \cup T_{i}$. Для каждого $i$ имеем: $E_{i} \subseteq\left\{e_{1}, \ldots, e_{i}\right\}$. Элемент схемы помещается в множество $E_{i}$ на $i$-м шаге, если его входы не присоединены ко входам схемы, свободным относительно множества $F_{i} \cup T_{i}$.

Отметим, что с ростом номера $i$ множества $F_{i}, T_{i}$ и $E_{i}$ не уменьшаются. В начале построения полагаем $i=0, F_{0}=T_{0}=\varnothing, E_{0}=\varnothing$ и, как уже было сказано, $C=\{\mathbf{0}, \mathbf{1}\}$.

Определим основное понятие, на котором основано рассуждение.

Определение 1. Пусть задано множество элементов $E \subseteq\left\{e_{1}, \ldots, e_{s}\right\}$. Подадим на входы схемы $S$ произвольный набор $\boldsymbol{\alpha} \in\{0,1\}^{n}$. Пусть $e_{r_{1}}, \ldots, e_{r_{l}}-$ это все такие элементы схемы, что для всякого $j \in[l]$ :

1) $h_{r_{j}}(\boldsymbol{\alpha})=1$;

2) $e_{r_{j}} \in\left\{e_{1}, \ldots, e_{s}\right\} \backslash E$.

Элемент $e_{m}$, обладающий минимальным номером среди элементов $e_{r_{1}} \ldots, e_{r_{l}}$, называется первым ненулевым элементом на наборе $\boldsymbol{\alpha}$ относительно множества $Е$.

Иногда, говоря о первом ненулевом элементе на некотором наборе, мы не будем указывать явно, относительно какого множества он рассматривается, так как это будет понятно из контекста.

Будем строить цепь таким образом, чтобы для любого $i$ после $i$-го шага имели место следующие свойства.

(1) Для всех $t, p \leqslant i: F_{t} \cap T_{p}=\varnothing$ (т. е. нельзя зафиксировать переменную, например, единицей, а позже - нулем; неформально об этом свойстве говорилось ранее).

(2) Для всякого элемента $e_{j} \in\left\{e_{1}, \ldots, e_{i}\right\} \backslash E_{i}$ и для функции $h_{j}$, которая вычисляется на выходе элемента $e_{j}$, выполнено равенство

$$
h_{j}\left(\boldsymbol{x}^{[n] \backslash F_{i}}\right)=h_{j}\left(\boldsymbol{x}^{T_{i}}\right)=0 .
$$

Свойство 2 - ключевое в процессе построения цепи. Неформально говоря, оно означает, что после $i$-го шага при подаче на входы схемы верхнего и нижнего наборов текущего подкуба все элементы вплоть до $i$-го, кроме элементов из множества $E_{i}$, выдают на этих наборах 0.

Процедура построения цепи разбивается на два этапа.

Этап I. Этап состоит из $s$ шагов по числу элементов схемы. Предположим, что было выполнено $i$ шагов, опишем $(i+1)$-й шаг.

По окончании $i$-го шага рассмотрены первые $i$ элементов схемы: $e_{1}, e_{2}, \ldots, e_{i}$. Построено некоторое множество $F_{i}$, содержащее номера входных переменных схемы, зафиксированных значением 0. Также построено множество $T_{i}$, содержащее номера входных переменных схемы, зафиксированных значением 1. Цепь $C$ содержит наборы $\mathbf{0 , 1} \in\{0,1\}^{n}$, а также наборы, полученные одновременно с построением множеств $F_{i}$ и $T_{i}$. Построено множество элементов $E_{i}$. Рассматривается подкуб размерности $n-\left|F_{i}\right|-\left|T_{i}\right|$ с верхним и нижним наборами $\boldsymbol{x}^{[n] \backslash F_{i}}$ и $\boldsymbol{x}^{T_{i}}$, соответственно.

Итак, начиная $(i+1)$-й шаг, переходим к элементу $e_{i+1}$. Возможны следующие случаи (отметим, что они не могут реализоваться одновременно). 
(1) На входы элемента $e_{i+1}$ не подаются непосредственно входы схемы, свободные относительно множества $F_{i} \cup T_{i}$. Тогда полагаем $E_{i+1}=E_{i} \cup\left\{e_{i+1}\right\}, F_{i+1}=F_{i}$, $T_{i+1}=T_{i}$. На этом $(i+1)$-й шаг закончен.

(2) На какой-либо вход элемента $e_{i+1}$ непосредственно подается хотя бы один из входов схемы, свободный относительно множества $F_{i} \cup T_{i}$. Пусть этому входу приписана некоторая переменная $x_{m}$, где $m \in[n] \backslash\left(F_{i} \cup T_{i}\right)$. Проверим, выполнено ли свойство 2 для элемента $e_{i+1}: h_{i+1}\left(\boldsymbol{x}^{[n] \backslash F_{i}}\right)=h_{i+1}\left(\boldsymbol{x}^{T_{i}}\right)=0$.

2.1. Если свойство 2 выполнено, то полагаем $E_{i+1}=E_{i}, F_{i+1}=F_{i}, T_{i+1}=T_{i}$, и $(i+1)$-й шаг закончен.

2.2. Пусть свойство 2 для $e_{i+1}$ не выполнено на верхнем наборе подкуба: $h_{i+1}\left(\boldsymbol{x}^{[n] \backslash F_{i}}\right)=1$ (случай нижнего набора будет разобран позже). Для удобства разобьем изложение этого случая на два подэтапа.

2.2.1. Вначале мы работаем с элементом $e_{i+1}$. Из условия случая 2.2 следует, что элемент $e_{i+1}$ является первым ненулевым на наборе $\boldsymbol{x}^{[n] \backslash F_{i}}$ относительно множества $E_{i}$. Зафиксируем значение указанной выше переменной $x_{m}$ нулем.

Будем использовать вспомогательные обозначения: $F_{i+1}^{r}, E_{i+1}^{r}$ и, в дальнейшем, $T_{i+1}^{r}$, где $r$ - натуральный параметр.

Для $r=1$ полагаем $F_{i+1}^{1}=F_{i} \cup\{m\}$; также полагаем $T_{i+1}=T_{i}$. Тем самым, мы переходим к подкубу размерности на единицу меньше (размерности $n-\left|F_{i+1}^{1}\right|-\left|T_{i+1}\right|$ ). Добавляем в цепь $C$ верхний набор этого подкуба $-\boldsymbol{x}^{[n] \backslash F_{i+1}^{1}}$. Положим множество $E_{i+1}^{1}$ равным объединению множества $E_{i}$ и множества всех элементов $e_{j}$, где $j \leqslant i+1$, на входы которых после фиксирования переменной $x_{m}$ более не подаются непосредственно входы схемы, свободные относительно множества $F_{i+1}^{1} \cup T_{i+1}$.

2.2.2. Далее мы работаем с элементами $e_{j}$, где $j \leqslant i+1$. Вначале проведем рассуждение, по-прежнему считая значение $r$ равным 1 .

Рассмотрим элементы $e_{j}$, где $j \leqslant i+1$ и $e_{j} \notin E_{i+1}^{r}$, и проверим, выполнено ли для них свойство 2 на наборе $\boldsymbol{x}^{[n] \backslash F_{i+1}^{r}}$. Пусть нашлись элементы, для которых свойство 2 не выполнено, и пусть $e_{l}$ - элемент с минимальным номером среди них.

Лемма 3. Хотя бъ на один из входов указанного элемента е дается непосредственно какой-либо вход схемы, свободный относительно множества $F_{i+1}^{r} \cup T_{i+1}$.

Доказательство. Для функции $h_{l}$, которая реализуется на выходе элемента $e_{l}$, имеем: $h_{l}\left(\boldsymbol{x}^{[n] \backslash F_{i+1}^{r}}\right)=1, h_{l}\left(\boldsymbol{x}^{T_{i+1}}\right)=0$. Значение антицепной функции $g_{l}$, соответствующей элементу $e_{l}$, определяется значениями на некоторых входах схемы, а также значениями на выходах элементов двух типов:

1) таких элементов $e_{u}$, что $e_{u} \notin E_{i+1}^{r}$, где $u<l$,

2) таких элементов $e_{u}$, что $e_{u} \in E_{i+1}^{r}$, где $u<l$.

Значения на выходах элементов 2-го типа определяются значениями на выходах элементов 1-го типа. В силу минимальности $l$ все элементы $e_{j}$ 1-го типа на верхнем и нижнем наборах подкуба выдают 
0. Следовательно, значения на выходах элементов 2-го типа на указанных двух наборах одинаковы. Получаем, что функция $g_{l}$ выдает различные значения на некоторой паре наборов со следующим свойством: в этих наборах компоненты, соответствующие значениям на выходах элементов с меньшими номерами, совпадают. Следовательно, значения каких-то из оставшихся компонент в этих наборах должны различаться. Таким образом, хотя бы один из входов схемы, свободный относительно множества $F_{i+1}^{r} \cup T_{i+1}$, подается непосредственно на один из входов элемента $e_{l}$. Лемма 3 доказана.

Продолжим основное рассуждение. По лемме 3 получаем, что элемент $e_{l}$ является первым ненулевым элементом на наборе $\boldsymbol{x}^{[n] \backslash F_{i+1}^{r}}$ относительно множества $E_{i+1}^{r}$. Рассмотрим произвольный вход схемы, свободный относительно множества $F_{i+1}^{r} \cup T_{i+1}$, который подается непосредственно на некоторый вход элемента $e_{l}$. Зафиксируем переменную, которая приписана этому входу схемы, значением 0. Тем самым, мы добавим номер этой входной переменной в множество $F_{i+1}^{r}$. Обозначим полученное множество через $F_{i+1}^{r+1}$. Мы вновь перешли к подкубу размерности на единицу меньше. Верхний набор этого подкуба $\boldsymbol{x}^{[n] \backslash F_{i+1}^{r+1}}$ добавляем в цепь $C$.

Полагаем множество $E_{i+1}^{r+1}$ равным объединению множества $E_{i+1}^{r}$ и множества всех элементов $e_{j}$, где $j \leqslant i+1$, на входы которых после фиксирования указанной выше переменной более не подаются непосредственно входы схемы, свободные относительно множества $F_{i+1}^{r+1} \cup T_{i+1}$.

Далее мы действуем по циклу: вновь выполняем процесс, описанный в п. 2.2.2, для всех $r=2, \ldots, q$, где $q$ - это такое значение параметра $r$, при котором свойство 2 будет выполнено для всех элементов $e_{j}$, где $j \in[i+1]$ и $e_{j} \notin E_{i+1}^{q}$.

Отметим важное свойство, которое потребуется в дальнейшем.

Лемма 4. Любой элемент $e_{p}$, где $p \in[i+1]$, добавленный в множество $E_{i+1}^{r}$ c $r \leqslant q$, в рамках описанного в $n .2 .2$ прочесса выдает 0 на всех наборах подкуба размерности $n-\left|F_{i+1}^{r}\right|-\left|T_{i+1}\right|$, на которых элементы $e_{a}$, где $a<p, e_{a} \notin E_{i+1}^{r}$, выдают 0 .

Доказательство. На входы элемента $e_{p}$ не подаются входы схемы, свободные относительно множества $F_{i+1}^{r} \cup T_{i+1}$, поэтому на всех указанных наборах этот элемент выдает одно и то же значение. На нижнем наборе подкуба он выдает 0 (так как $T_{i+1}=T_{i}$ ). Значит, на всех описанных наборах подкуба он также выдает 0 . Лемма 4 доказана.

После того, как описанные действия выполнены, полагаем $F_{i+1}=F_{i+1}^{q}$, $E_{i+1}=E_{i+1}^{q}$, и $(i+1)$-й шаг закончен.

Рассмотрим оставшийся случай.

2.3. Пусть свойство 2 для $e_{i+1}$ не выполнено на нижнем наборе подкуба: $h_{i+1}\left(\boldsymbol{x}^{T_{i}}\right)=1$.

Лемма 5. Случаи 2.2 и 2.3 несовместимы. 
Доказательство. Предположим противное. При этом имеем: для всех $e_{j} \notin E_{i}$, где $j<i+1$, функции $h_{j}$ на наборах $\boldsymbol{x}^{[n] \backslash F_{i}}$ и $\boldsymbol{x}^{T_{i}}$ выдают 0; для всех $e_{j} \in E_{i}$, где $j<i+1$, функции $h_{j}$ на этих наборах выдают одно и то же значение. Наборы $\boldsymbol{x}^{[n] \backslash F_{i}}$ и $\boldsymbol{x}^{T_{i}}$ сравнимы, и, по условию случая 2.2 , какой-либо из входов элемента $e_{i+1}$ присоединен ко входу схемы, на котором на этих наборах будут различные значения. Значит, функция $g_{i+1}$ выдает 1 на некоторой паре сравнимых наборов, отличающихся по крайней мере в одной компоненте. Получаем противоречие с тем, что $g_{i+1}$ - антицепная функция. Лемма 5 доказана.

Итак, вернемся к случаю 2.3: он двойствен случаю 2.2, и мы действуем аналогично, с той лишь разницей, что входные переменные фиксируются значением 1. Итак, вначале зафиксируем указанную в п. 2 переменную $x_{m}$ единицей и положим $T_{i+1}^{1}=T_{i} \cup\{m\}$ (следуя обозначениям, введенным в 2.2). Положим $F_{i+1}=F_{i}$. Таким образом, переходим к подкубу размерности $n-\left|F_{i+1}\right|-\left|T_{i+1}^{1}\right|$. Помещаем в цепь $C$ набор $\boldsymbol{x}^{T_{i+1}^{1}}$. Далее, аналогично случаю 2.2 , выполняем цикл, описанный в 2.2 .2 , получая, соответственно, множества $T_{i+1}^{2}, T_{i+1}^{3}, \ldots$ до тех пор, пока для некоторого $v$ свойство 2 не будет выполнено для всех таких элементов $e_{j}$, что $j \leqslant i+1$ и $e_{j} \notin E_{i+1}^{v}$. После этого полагаем $T_{i+1}=T_{i+1}^{v}, E_{i+1}=E_{i+1}^{v}$, и $(i+1)$-й шаг закончен.

Если в результате выполнения шагов этапа I зафиксировали значения всех входных переменных, то цепь, состоящая из $n+1$ наборов, построена, и процесс заканчивается.

Пусть мы совершили $s$ шагов, описанных в случаях 1 и 2 этапа I, т. е. рассмотрели последовательно все элементы схемы $S$, однако искомая цепь еще не построена. После $s$-го шага получены множества $F_{s}, T_{s}$ и $E_{s}$ - для краткости далее опустим индексы и будем писать $F, T$ и $E$, соответственно. Переходим к этапу II.

Этап II. Исходный куб размерности $n$ сужен до подкуба размерности $n-|F|-|T|$ так, что все элементы схемы $e_{1}, \ldots, e_{s}$, кроме элементов из $E$, выдают 0 на верхнем и нижнем наборах этого подкуба $-\boldsymbol{x}^{[n] \backslash F}$ и $\boldsymbol{x}^{T}$. Положим $T^{0}=T$. Будем добавлять в $T^{0}$ номера переменных из $[n] \backslash(F \cup T)$, получая множества $T^{1}, T^{2}$ и т. д., следующим образом:

1) если $i \geqslant 0$ и на наборе $\boldsymbol{x}^{T^{i}}$ нет первого ненулевого элемента относительно множества $E$, то добавляем в множество $T^{i}$ номер произвольной переменной из множества $[n] \backslash\left(F \cup T^{i}\right)$. Получаем множество $T^{i+1}$ и набор $\boldsymbol{x}^{T^{i+1}}$, который помещаем в цепь;

2) если $i \geqslant 0$ и на наборе $\boldsymbol{x}^{T^{i}}$ есть первый ненулевой элемент относительно множества $E$, то аналогично доказательству леммы 3 нетрудно показать, что какой-либо из входов этого элемента присоединен ко входу схемы, свободному относительно множества $[n] \backslash\left(F \cup T^{i}\right)$. Зафиксируем единицей входную переменную, которая соответствует этому входу. Получаем множество $T^{i+1}$ и набор $\boldsymbol{x}^{T^{i+1}}$, который помещаем в цепь.

Если в рамках описанного процесса при добавлении номера какой-либо переменной в множество $T^{i}$ входы некоторого элемента $e_{d}$ более не присоединены ко входам схемы, свободным относительно множества $F \cup T^{i}$, то полагаем $E=E \cup\left\{e_{d}\right\}$. Нетруд- 
но понять, что все такие элементы обладают свойством, аналогичным указанному в лемме 4. Отметим, что первый ненулевой элемент рассматривается всякий раз относительно текущего множества $E$.

Повторяем описанный процесс до тех пор, пока все входные переменные не будут зафиксированы, т. е., пока не будет построена искомая цепь $C$. Отметим, что на этапе 2 множество $T$ выбрано для определенности: можно было выбрать множество $F$ и, соответственно, фиксировать переменные значением 0.

При построении цепи $C$ на входы схемы подаются только наборы $\boldsymbol{x}^{T}$ и $\boldsymbol{x}^{[n] \backslash F}$ (для краткости индексы опущены). Эти наборы сравнимы, так как на каждом шаге $T \subseteq[n] \backslash F$. Таким образом, множество $C$ действительно является цепью.

3.3. Завершение доказательства леммы 1. Цепь $C$ обладает следующим свойством.

Лемма 6. Никакой элемент схемы $S$ не оказывается первым ненулевым элементом (относительно соответствующих множеств) на двух различных наборах цепи $C$ в процессе ее построения.

Доказательство. Будем вести доказательство методом «от противного». Пусть, без ограничения общности, набор $\boldsymbol{x}^{P}$ добавлен в цепь на шаге с номером $b$, а $\boldsymbol{x}^{P^{\prime}}$ на шаге с номером $c$, где $b<c$. На этих шагах были построены множества $E_{b}$ и $E_{c}$, причем $E_{b} \subseteq E_{c}$. И пусть существует такой $t \in[s]$, что элемент $e_{t}-$ первый ненулевой элемент на наборах $x^{P}$ и $x^{P^{\prime}}$ относительно соответствующих множеств. В частности, $h_{t}\left(\boldsymbol{x}^{P}\right)=h_{t}\left(\boldsymbol{x}^{P^{\prime}}\right)=1$. Для всякого элемента $e_{j} \notin E_{c}$, где $j<t$, по определению первого ненулевого элемента (относительно множества $E_{c}$, а следовательно, и $\left.E_{b}\right)$, выполнено равенство: $h_{j}\left(\boldsymbol{x}^{P}\right)=h_{j}\left(\boldsymbol{x}^{P^{\prime}}\right)=0$. Также для всякого элемента $e_{j} \in E_{c} \backslash E_{b}$, где $j<t$, по свойству из леммы 4 имеем: $h_{j}\left(\boldsymbol{x}^{P}\right)=h_{j}\left(\boldsymbol{x}^{P^{\prime}}\right)=0$, а значения элементов $e_{j} \in E_{b}$, где $j<t$, определяются значениями остальных элементов с меньшими номерами. Следовательно, значения всякого элемента с номером меньше $t$ одинаковы на наборах $\boldsymbol{x}^{P^{\prime}}$ и $\boldsymbol{x}^{P}$. При этом, по построению, набор $\boldsymbol{x}^{P^{\prime}}$ отличается от набора $\boldsymbol{x}^{P}$ значением хотя бы одной компоненты, соответствующей входной переменной схемы, непосредственно подаваемой на один из входов элемента $e_{t}$.

Таким образом, для антицепной функции $g_{t}$, соответствующей элементу $e_{t}$, имеем: $g_{t}$ выдает 1 на некоторой паре сравнимых наборов. Это противоречит тому, что $g_{t}$ - антицепная функция. Лемма 6 доказана.

Завершим доказательство леммы 1 . Напомним, что построенная цепь $C$ содержит $n+1$ наборов куба $\{0,1\}^{n}$. На всех наборах цепи, на которых в схеме $S$ нет первого ненулевого элемента относительно соответствующего множества, значение на выходе схемы одинаковое, обозначим его через $a$, где $a \in\{0,1\}$. На любом наборе цепи, на котором схема выдает $1-a$, есть первый ненулевой элемент. Функция $f\left(x_{1}, \ldots, x_{n}\right)$ принимает значение 1 на $k(f)$ наборах этой цепи, а значение $0-$ на $n+1-k(f)$ наборах. Возможны следующие случаи.

1. Если $a=0$, то для любого набора, на котором схема выдает 1 , в схеме есть первый ненулевой элемент. То есть на $k(f)$ наборах цепи $C$ есть первые ненулевые элементы. По лемме 6 все эти элементы различны. Отсюда получаем, что $L(S) \geqslant$ $k(f)$. 
2. Если $a=1$, то для любого набора, на котором схема выдает 0, в схеме есть первый ненулевой элемент. То есть на $n+1-k(f)$ наборах цепи $C$ есть первые ненулевые элементы. Аналогично, по лемме 6, получаем: $L(S) \geqslant n+1-k(f)$. При этом последний элемент схемы (элемент $e_{s}$ ) не является первым ненулевым так как если на некотором наборе в схеме есть первый ненулевой элемент, то, по условию случая, схема выдает 0, т. е. элемент $e_{s}$ выдает 0. Отсюда вытекает, что $L(S) \geqslant n+2-k(f)$.

Итак, мы показали, что $L(S) \geqslant \min (k(f), n-k(f)+2)$. В силу произвольности схемы $S$, получаем аналогичное неравенство для величины $L(f)$. Лемма 1 полностью доказана.

3.4. Доказательство леммы 2. Рассмотрим произвольную симметрическую функцию $f\left(x_{1}, \ldots, x_{n}\right)$. Разобьем доказательство на две части: в первой части докажем оценку $L(f) \leqslant k(f)$, а во второй - оценку $L(f) \leqslant n-k(f)+2$. Вместе эти оценки дают требуемое утверждение.

Доказательство. (1) Пусть функция $f$ равна 1 на слоях с номерами $i_{1}, \ldots, i_{k(f)}$, причем $i_{1}<\ldots<i_{k(f)}$.

Напомним, что набор значений аргументов $\left(x_{1}, \ldots, x_{n}\right)$ обозначается через $\boldsymbol{x}$. Для функции $f$, для каждого $t \in\left\{i_{1}, \ldots, i_{k(f)}\right\}$ зададим функцию $h_{t}(\boldsymbol{x})$ так: $h_{t}(\boldsymbol{x})=1$ тогда и только тогда, когда $\sum_{p=1}^{n} x_{p}=i_{t}$. Ясно, что функции $h_{t}$ - антицепные, поскольку являются характеристическими функциями слоев куба.

Обозначим через $\boldsymbol{y}$ набор значений аргументов $\left(y_{1}, \ldots, y_{k(f)-1}\right)$.

Пусть $M_{1}-$ множество таких наборов $(\boldsymbol{y}, \boldsymbol{x})$, что одновременно выполнены три условия: 1) существует такой номер $j \in[k(f)-1]$, что $y_{j}=1$; 2$) y_{q}=$ 0 для всех $q \neq j ; 3) \sum_{p=1}^{n} x_{p}=i_{j}$. Пусть $M_{2}-$ множество наборов $(\boldsymbol{y}, \boldsymbol{x})$, удовлетворяющих следующим условиям: $y_{q}=0$, и $\sum_{p=1}^{n} x_{p}=i_{k(f)}$ для всех $q \in[k(f)-1]$.

Зададим булеву функцию $g$ от $k(f)-1+n$ переменных $y_{1} \ldots, y_{k(f)-1}, x_{1} \ldots, x_{n}$ так: $g(\boldsymbol{y}, \boldsymbol{x})=1$ лишь на наборах $(\boldsymbol{y}, \boldsymbol{x})$ из множества $M_{1} \sqcup M_{2}$.

Нетрудно понять, что функция $g$ - антицепная. Действительно, рассмотрим два набора из носителя $g:\left(\boldsymbol{y}_{1}, \boldsymbol{x}_{1}\right) \neq\left(\boldsymbol{y}_{2}, \boldsymbol{x}_{2}\right)$. Если $\left(\boldsymbol{y}_{1}, \boldsymbol{x}_{1}\right),\left(\boldsymbol{y}_{2}, \boldsymbol{x}_{2}\right) \in M_{1}$ и $\boldsymbol{y}_{1} \neq \boldsymbol{y}_{2}$, то наборы не сравнимы. Если $\left(\boldsymbol{y}_{1}, \boldsymbol{x}_{1}\right),\left(\boldsymbol{y}_{2}, \boldsymbol{x}_{2}\right) \in M_{1}$ и $\boldsymbol{y}_{1}=\boldsymbol{y}_{2}$, то номера компонент, в которых стоят по $i_{j}$ единиц в наборах $\boldsymbol{x}_{1}$ и $\boldsymbol{x}_{2}$, не совпадают. А значит, наборы $\left(\boldsymbol{y}_{1}, \boldsymbol{x}_{1}\right)$ и $\left(\boldsymbol{y}_{2}, \boldsymbol{x}_{2}\right)$ не сравнимы. Если $\left(\boldsymbol{y}_{1}, \boldsymbol{x}_{1}\right),\left(\boldsymbol{y}_{2}, \boldsymbol{x}_{2}\right) \in M_{2}$, то, аналогично предыдущему случаю, легко видеть, что наборы несравнимы. Если, без ограничения общности, $\left(\boldsymbol{y}_{1}, \boldsymbol{x}_{1}\right) \in M_{1},\left(\boldsymbol{y}_{2}, \boldsymbol{x}_{2}\right) \in M_{2}$, то $\boldsymbol{y}_{1}>\boldsymbol{y}_{2}$, а в $\boldsymbol{x}_{2}$ больше единиц, чем в $\boldsymbol{x}_{1}$. Значит, наборы $\left(\boldsymbol{y}_{1}, \boldsymbol{x}_{1}\right),\left(\boldsymbol{y}_{2}, \boldsymbol{x}_{2}\right)$ несравнимы. Реализуем функцию $f(\boldsymbol{x})$ так:

$$
f(\boldsymbol{x})=g\left(h_{1}(\boldsymbol{x}), \ldots, h_{k(f)-1}(\boldsymbol{x}), \boldsymbol{x}\right) .
$$


То есть, если подставить в функцию $g$ характеристические функции слоев булева куба, образующих в совокупности носитель функции $f-$ все, кроме функции $h_{k(f)}$, - а также переменные, то получится реализация функции $f$. Проверим равенство (1). Рассмотрим произвольный набор $\boldsymbol{\alpha}=\left(\alpha_{1}, \ldots, \alpha_{n}\right)$. Возможны два случая.

А. Если $f(\boldsymbol{\alpha})=1$, то $\sum_{p=1}^{n} \alpha_{p} \in\left\{i_{1}, \ldots, i_{k(f)}\right\}$. Если $\sum_{p=1}^{n} \alpha_{p} \leqslant i_{k(f)-1}$, то существует такой номер $j \in[k(f)-1]$, что $h_{j}(\boldsymbol{\alpha})=1$ и $h_{q}(\boldsymbol{\alpha})=0$ и $\sum_{p=1}^{n} \alpha_{p}=i_{j}$ для любого $q \neq j$. Получаем, что набор $(\boldsymbol{y}, \boldsymbol{\alpha})$, где $\left(y_{1}, \ldots, y_{k(f)-1}, \boldsymbol{\alpha}\right)=\left(h_{1}(\boldsymbol{\alpha}), \ldots, h_{k(f)-1}(\boldsymbol{\alpha}), \boldsymbol{\alpha}\right)$, принадлежит множеству $M_{1}$. Значит, $g(\boldsymbol{y}, \boldsymbol{\alpha})=1$. Если $\sum_{p=1}^{n} \alpha_{p}=i_{k(f)}$, то $y_{q}=h_{q}(\boldsymbol{\alpha})=0$ для любого $q \in[k(f)-1]$. Таким образом, набор $(\boldsymbol{y}, \boldsymbol{\alpha})$ принадлежит $M_{2}$, и следовательно, $g(\boldsymbol{y}, \boldsymbol{\alpha})=1$.

В. Если $f(\boldsymbol{\alpha})=0$, то $h_{j}(\boldsymbol{\alpha})=0$ для любого $j \in[k(f)]$. В частности, $h_{k(f)}(\boldsymbol{\alpha})=0$, а значит, $\sum_{p=1}^{n} \alpha_{p} \neq i_{k(f)}$. Отсюда получаем, что набор $(\boldsymbol{y}, \boldsymbol{\alpha})=\left(h_{1}(\boldsymbol{\alpha}), \ldots, h_{k(f)-1}(\boldsymbol{\alpha}), \boldsymbol{\alpha}\right)$ не принадлежит ни $M_{1}$, ни $M_{2}$. Следовательно, $g(\boldsymbol{y}, \boldsymbol{\alpha})=0$.

Из (1) следует, что функция $f$ может быть реализована схемой в базисе $A C$ со сложностью не больше $k(f)$.

Получаем, что для всякой симметрической функции $f$ существует схема $S_{f}$ сложности $L\left(S_{f}\right) \leqslant k(f)$, которая реализует функцию $f$ и обладает следующим свойством: на входы всякого элемента схемы подаются все ее входы.

(2) Из доказательства для случая 1 получаем, что для функции $\bar{f}$ выполнено неравенство $L(\bar{f}) \leqslant k(\bar{f})$, где $k(\bar{f})=n+1-k(f)$. Таким образом, функция $\bar{f}$ может быть реализована схемой сложности не больше $n+1-k(f)$. Из любой схемы для этой функции с помощью элемента отрицания получается схема для функции $f$, то есть $L(f) \leqslant n-k(f)+2$.

Итак, для произвольной симметрической функции $f$ мы показали, что $L(f) \leqslant$ $\min (k(f), n-k(f)+2)$. Тем самым лемма 2 доказана.

Доказательства лемм 1 и 2 дают в совокупности доказательство теоремы 1.

\section{4. Доказательство теоремы 2}

Для функции голосования $k\left(m_{n}\right)=\left\lfloor\frac{n}{2}\right\rfloor+1$, по теореме 1 получаем: $L\left(m_{n}\right)=\left\lfloor\frac{n}{2}\right\rfloor+1$. Для функции четности $k\left(p_{n}\right)=\left\lfloor\frac{n+1}{2}\right\rfloor$, соответственно, по теореме 1 получаем: $L\left(p_{n}\right)=\left\lfloor\frac{n+1}{2}\right\rfloor$, что и доказывает теорему 2 .

Отметим, что максимум верхней оценки из леммы 2 достигается: при нечетных $n$ он равен сложности функций $m_{n}, p_{n}$ и $\bar{p}_{n}$, а при четных $n-$ сложности функций $m_{n}$ и $\bar{p}_{n}$.

Автор выражает глубокую признательность О. М. Касим-Заде за постановку задачи и постоянное внимание к работе. Автор благодарит также А. В. Кочергина за ценные замечания. 


\section{Список литературы}

1. Касим-Заде О. М., "О сложности схем в одном бесконечном базисе”, Вестник Московского Университета. Сер. 1. Матем. Мех., 1994, № 6, 40-44.

2. Лупанов О. Б., Асимптотические оченки сложности управляющих систем, Изд-во Московского ун-та, Москва, 1984, 138 с.

3. Касим-Заде О. М., "О сложности реализации булевых функций схемами в одном бесконечном базисе", Дискретный анализ и исследование операчий, 2:1 (1995), 7-20.

4. Подольская О. В., "О нижних оценках сложности схем в базисе антицепных функций", Вестник Московского Университета. Сер. 1. Матем. Мех., 2013, № 2, 17-23.

5. Подольская О. В., "Об оценках сложности схем в одном бесконечном базисе", Материалы IX Молодежной научной школы по дискретной математике и ее приложениям, Москва, 16-23 сентября, 2013, 97-100.

6. Лупанов О. Б., "Об одном методе синтеза схем”, Известия высших учебных заведений. Радиофизика, 1:1 (1958), 120-140.

Статья поступила 16.02.2015. 